\title{
Impact of coronavirus disease 2019 (COVID-19) emergency on Italian radiologists: a national survey
}

\author{
Domenico Albano ${ }^{1,2}$ (D) Antonio Bruno ${ }^{3}$. Federico Bruno ${ }^{4} \cdot$ Marco Calandri $^{5,6}$. Damiano Caruso ${ }^{7}$. \\ Alfredo Clemente ${ }^{8}$. Pietro Coppolino ${ }^{9}$. Diletta Cozzi ${ }^{10}$. Riccardo De Robertis ${ }^{11}$. Francesco Gentili ${ }^{12}$. \\ Irene Grazzini ${ }^{13}$. Maria Laura Jannone ${ }^{14}$. Carlo Liguori ${ }^{15}$. Raffaele Natella ${ }^{8}$. Genny Pace ${ }^{16}$. Alessandro Posa ${ }^{17}$. \\ Paola Scalise ${ }^{18} \cdot$ Bruno Accarino $^{19} \cdot$ Corrado Bibbolino $^{20} \cdot$ Antonio Barile $^{4} \cdot$ Roberto Grassi $^{8} \cdot$ Carmelo Messina $^{1,21} \cdot$ On \\ behalf of the Young SIRM Working Group
}

Received: 12 May 2020 / Revised: 5 June 2020 / Accepted: 25 June 2020 / Published online: 14 July 2020

(C) European Society of Radiology 2020

\begin{abstract}
Objectives To perform an online survey aimed at evaluating the impact of COVID-19 on Italian radiology departments. Methods We launched a survey composed of 25 questions about how COVID-19 has changed the safety and organization of daily activity in Italian radiology units.

Results A total of 2136/10,564 (20.2\%) radiologists of the Italian Society of Medical and Interventional Radiology participated. Two-thirds performed at least one diagnostic/interventional procedure on COVID-19 patients. The $88.1 \%$ reported a reduction in the elective imaging volumes, with US, mammography, and MRI having shown the greater decrease $(41.1 \%, 23.9 \%$, and $21.1 \%$, respectively). In $69.6 \%$ of cases, institutions had trouble getting personal protective equipment (PPE), especially public hospitals and southern institutions. Less than $30 \%$ of participants were subjected to RT-PCR swab test, although $81.5 \%$ believed that it should be done on all health workers and $70 \%$ suggested it as the most important measure to improve safety at work. Slightly more than half of participants declared to work safely and felt to be adequately protected by their institutions. Up to $20 \%$ of northern participants were redeployed to clinical services. The first imaging examination performed by admitted COVID-19 patients was chest radiography in $76.3 \%$ of cases. Almost half of participants reported that less than $30 \%$ of health workers were infected in their radiology department, with higher rates in northern regions and public institutions.

Conclusions This snapshot of the current situation in Italian radiology departments could be used to harmonize the organization of working activity in order to safely and effectively face this pandemic.

Key Points

- More than two-thirds of institutions had trouble getting PPE for health workers, with public hospitals and southern institutions that presented more procurement problems

- A substantial drop of imaging volumes was observed in the vast majority of Italian radiology departments, mostly due to the decrease of ultrasound, mammography, and MRI, especially in private practice were working activity was stopped in $13.3 \%$ of institutions

- RT-PCR swab to health workers was reported as the most suggested measure by Italian radiologists to improve safety at work, as more than $80 \%$ of them believed that it should be performed to all health workers, although less than $30 \%$ were subjected to this test
\end{abstract}

Keywords Pandemic $\cdot$ Coronavirus $\cdot$ COVID-19 $\cdot$ Survey $\cdot$ Teleradiology

Electronic supplementary material The online version of this article (https://doi.org/10.1007/s00330-020-07046-7) contains supplementary material, which is available to authorized users.

Domenico Albano

albanodomenico@me.com

Extended author information available on the last page of the article

\author{
Abbreviations \\ C O V I D -Coronavirus disease 2019 \\ 19 \\ PPE Personal protective equipment \\ RT-PCR Reverse transcriptase-polymerase chain reaction \\ SIRM Italian Society of Medical and Interventional \\ Radiology
}




\section{Introduction}

The coronavirus disease 2019 (COVID-19) infection caused by a novel type of coronavirus (SARS-CoV-2) has been declared as a pandemic by the World Health Organization on March 11, due to the rapid increase in the number of cases worldwide [1]. Since the outbreak of COVID-19, Italy has been the epicenter of the infection in Europe, with the highest number of deaths as of May 1, 2020 (27,967 deaths, globally second only to the USA) [2-4].

Immediately after the first cases on February 2020, Italy Government established containment social distancing measures, ultimately leading to the national lockdown which was imposed from 9 March until 4 May 2020 [5]. This dramatic scenario forced hospitals to undertake extreme measures to change daily activity organization, to optimize the management of COVID-19 as well as non-COVID-19 patients [6]. Among the others, these measures included hospital access restrictions, cessation of elective activities, the identification of dedicated COVID-19 isolation pathways, and the allocation of COVID-19 patients to non-emergency hospital wards [5, $6]$.

Radiology departments play an essential role in the management of COVID-19 patients, due to the irreplaceable diagnostic role of CT and x-ray scans [7]. At the same time, radiology services are a crossroad of heterogeneous subjects within hospitals; thus, they are primarily involved in the triage of suspected patients as well as in staff protection [8]. In Italy, due to the recent explosion of new cases, patient categorization into suspect or confirmed COVID-19 and non-COVID19 has become challenging, with the concrete scenario of considering any subject coming into the radiology department as a possible COVID-19 case [9].

In Italy, the geographical distribution of the infection was uneven, with the northern regions that face disproportionately higher numbers of contagions and deaths compared with the central and southern regions [10]. To support Italian radiologists in becoming familiar with COVID-19 imaging features, the Italian Society of Medical and Interventional Radiology (SIRM) established a dedicated page on the SIRM website with an Italian collection of COVID-19 exams [11]. Also, SIRM provided operational procedures to help radiology units in the daily management of COVID-19 patients [12]. Nevertheless, due to the regional variability in COVID-19 distribution, certain variability may be expected regarding prevention and control measures in the Italian radiology departments. Therefore, SIRM with the cooperation of its Young Group promoted a national survey aimed at evaluating the impact of COVID-19 on radiology units.

This paper presents the results of this survey, providing a full report of which measures were instituted to control the infection spreading, and what was the impact on daily operational practices.

\section{Materials and methods}

\section{Study design}

Institutional Review Board approval was not needed for this article, as no patients were involved.

The Young SIRM Working Group launched a national survey to get national data about how COVID-19 pandemic has changed the safety and organization of working activity in Italian radiology departments, as well as which measures were adopted to face the emergency. Similar to previous studies, the questionnaire was created and distributed using the free online tool "Google Forms" (Google LLC) [13-15]. The survey was approved by the SIRM Board Committee on April 9, 2020, and an email was sent out to all 10,564 SIRM members on April 14. The participants were informed that questionnaire would have been managed in aggregated form to ensure anonymity. After 8 days, another email was sent out as a reminder. The survey was closed on April 30. During the entire opening period of the survey, Italy has been in the eye of the storm of COVID-19 emergency with the whole country on lockdown.

The questionnaire consisted of 25 questions: 23 closed answers with single $(n=16)$ or multiple-choice selections $(n=$ 7), plus 2 open questions with free text response. The list of questions and answers is reported in Table 1.

\section{Data analysis}

Data were collected and analyzed by two radiologists with more than 5 years of experience in medical research (D.A. and C.M.). The different answers given by participants under "other" choice (questions no. 13, no. 14, no. 15) were categorized into common headings (e.g., "no specific measures" and "don't know/no opinion"). We performed data sub-analysis according to the typology of healthcare delivery (public, private, and university institutions). Regional sub-analysis was performed by comparing answers from the three Italian macroregions (north, central, and south and Islands). We further analyzed data to highlight differences between the four northern Italian regions (Lombardia, Emilia-Romagna, Piemonte/Valle d'Aosta, Veneto/Trentino-Alto-Adige) with the highest infection rate. Indeed, when the survey was launched, these regions accounted for $75 \%$ of COVID-19 patients according to the Italian National Institute of Health (Fig. 1) [16, 17].

Data and response rates were expressed as mean \pm standard deviation and percentages. Comparisons between proportions were done using a chi-square test. The SPSS (v.26, IBM) was used for statistical analysis. A $p$ value $<0.05$ was considered statistically significant. 
Table 1 The full list of questions and answers

Question

1. Are you a certified radiologist or radiology resident?

2. What type of hospital do you work in?

3. Did you perform diagnostic or interventional procedures on patients with COVD-19?

4. Did you perform diagnostic or interventional procedures on patients with later diagnosis of COVD-19 for which you have been subsequently considered as exposed?

5. During COVID-19 emergency, your routinely working activity is:

6. During COVID-19 emergency, the working activity (diagnostic and interventional) of your institution has been targeted to:

7. Among the following radiological procedures, which one has shown the greatest decrease?

8. Did your institution have any trouble getting personal protective equipment (PPE)?

9. Which PPE did your institution provide to health workers? (multiple choice)

10. When did your institution perform RT-PCR swab on health workers?

11. In your opinion, when should health workers be subjected to RT-PCR swab?

12. Were you subjected to RT-PCR swab?
Answer

Certified radiologist: 1878 (87.9\%)

Resident: 258 (12.1\%)

Public hospital: 1095 (51.3\%)

University hospital: $432(20.2 \%)$

Private practice: 406 (19\%)

Accredited medical facility: 151 (7.1\%)

Local health service: 52 (2.4\%)

Yes: $1497(70.1 \%)$

No: 639 (29.9\%)

No: $907(42.5 \%)$

Yes: $687(32.2 \%)$

Not sure: $542(25.4 \%)$

Highly decreased: 1027 (48.1\%)

Decreased: 857 (40.1\%)

Stopped: $108(5.1 \%)$

Unchanged: $61(2.9 \%)$

Increased: $59(2.8 \%)$

Highly increased: 24 (1.1\%)

Emergency procedures and COVID-19 patients: 988 (46.3\%)

Elective procedures, emergency procedures and COVID-19 patients: 528 (24.7\%)

Only emergency procedures: $453(21.2 \%)$

Working activity has been stopped: $60(2.8 \%)$

Only COVID-19 patients: 54 (2.5\%)

Working activity has not changed: $53(2.5 \%)$

Ultrasound: $876(41 \%)$

Mammography: 510 (23.9\%)

MRI: $450(21.1 \%)$

Radiography: 146 (6.8\%)

Interventional procedures: 78 (3.7\%)

CT: $76(3.6 \%)$

Yes: $1487(69.6 \%)$

No: 649 (30.4\%)

Surgical face mask: $2026(94.9 \%)$

Gloves: 2013 (94.2\%)

Single use gowns: $1597(74.8 \%)$

FFP2/FFP3 face mask: 1260 (59\%)

Protective goggles $1090(51 \%)$

Visor: 956 (44.8\%)

On those with suspected COVID-19 symptoms: 817 (38.2\%)

After contact with COVID-19 patients: 670 (31.4\%)

All health workers were subjected to RT-PCR swabs: 325 (15.2\%)

Never: 324 (15.2\%)

RT-PCR swab should be performed on all health workers: 1740 (81.5\%) After contact with COVID-19/suspected COVID-19 patients: 291 (13.6\%) When health workers present suspected COVID-19 symptoms: 95 (4.4\%) Health workers should never be subjected to RT-PCR swabs: $10(0.5 \%)$

No: 1520 (71.2\%)

Yes: $616(28.8 \%)$ 
Table 1 (continued)

Question

13. Which measures did your institution/hospital adopt to face COVID-19 emergency? (multiple choice)

14. Which further measures did your radiology department adopt to face COVID-19 emergency? (multiple choice)

15. How do you think that safety of your work environment could be improved? (multiple choice)

16. Do you feel that you have been adequately protected by your institution during COVID-19 emergency?

17. Do you think you have been working safely during COVID-19 emergency?

18. Has your working activity been converted to support other specialists in COVID-19 departments/units?

19. Who is performing chest ultrasound in COVID-19/suspected covid-19 patients at your institution? (multiple choice)

20. Do you think that chest ultrasound: (multiple choice)
Answer

Different paths of COVID-19 and non-COVID-19 patients: 1335 (62.5\%)

Clinical and body temperature screening to all admitted patients and health workers: $1126(52.7 \%)$

Quick isolation of suspected COVID-19 patients: 1184 (55.4\%)

Clinical-anamnestic questionnaire to all admitted patients: 1094 (51.2\%)

Training courses for health workers on infection control and use of PPE: 799 $(37.4 \%)$

PPE to all admitted patients and health workers: 762 (35.7\%)

Centralization of supplies of PPE as needed: 601 (28.1\%)

Other: no specific measures $12(0.6 \%)$; interruption of activity: $11(0.5 \%)$; do not know/no opinion: 26 (1.2\%)

Increase availability of sanitizers and disinfectants: 1631 (76.4\%)

Interruption of routine examinations/procedures: 1571 (73.5\%)

Standardized protocols of sanitation of the examination rooms: 1489 (69.7\%)

Measures to ensure the interpersonal security distance: 1243 (58.2\%)

Use of radiology machines dedicated exclusively to COVID-19 patients: 988 $(46.2 \%)$

PPE to all patients prior to access the department or to perform examinations: $931(43.6 \%)$

Implementation of teleradiology activity: 203 (9.5\%)

Other: no specific measures 15 (0.7\%); do not know/no opinion: $22(1 \%)$

Increasing the number of swabs to reduce the infection among health workers: 1507 (70.5\%)

More PPE for health workers and patients: 1347 (63.1\%)

Improving the paths of patients: 1133 (53\%)

Managing COVID-19 patients in Institutions dedicated exclusively to these patients: $1102(51.6 \%)$

Providing PPE to all admitted patients and health workers: 1003 (47\%)

Using radiology machines dedicated exclusively to COVID-19 patients: 802 $(37.5 \%)$

Improving the training of health workers on infection control: 887 (41.5\%)

Improving teleradiology activity: 649 (30.4\%)

Other: serology tests: 13 (0.6\%); do not know/no opinion: $16(7.5 \%)$

Yes: $1169(54.7 \%)$

No: $967(45.3 \%)$

Yes: $1269(59.4 \%)$

No: $867(40.6 \%)$

No: $1849(86.6 \%)$

Yes: $287(13.4 \%)$

None: 919 (43\%)

Emergency physician: 639 (29.9\%)

Other specialists: 435 (20.4\%)

Pneumologist: 355 (16.6\%)

Internist: 319 (14.9\%)

Radiologist: 259 (12.1\%)

Should be done by an operator with experience in this technique: 1488 $(69.7 \%)$

Is useful: $707(33.1 \%)$

Is useless: $328(15.4 \%)$

Should be done by other specialists: 100 (4.7\%)

Chest radiography: 1629 (76.3\%) 
Table 1 (continued)

\begin{tabular}{ll}
\hline Question & Answer \\
\hline $\begin{array}{l}\text { 21. Which is the first imaging examination performed on } \\
\text { COVID-19 patients admitted to your institution? (multiple }\end{array}$ & Chest CT: $842(39.4 \%)$ \\
choice) & Chest ultrasound: $198(9.3 \%)$ \\
22. Has your knowledge in chest imaging been enough to face & Yes, but I broadened my knowledge: $1513(70.9 \%)$ \\
COVID-19 emergency? & Yes: $315(14.7 \%)$ \\
& No, but I broadened my knowledge: $308(14.4 \%)$ \\
23. Did any health worker in your radiology department get & No: $1189(55.7 \%)$ \\
COVID-19? & Yes, less than $10 \%$ of health workers: $768(36 \%)$ \\
& Yes, 10-30\% of health workers: $155(7.3 \%)$ \\
& Yes, 30-50\% of health workers: $18(0.8 \%)$ \\
24. How old are you (in years)? (open) & Yes, more than 50\% of health workers: $6(0.3 \%)$ \\
25. In which region do you work? (open) & Mean: $47 \pm 12$; range: $26-83$ years
\end{tabular}

COVID-19, coronavirus disease 2019; $P P E$, personal protective equipment; $R T-P C R$, reverse transcriptase-polymerase chain reaction

Fig. 1 Italian distribution of COVID-19 cases when the survey was officially closed nearing the end of lockdown (April 30, 2020, 205,463 confirmed cases). This figure is a derivative of "Map of provinces with confirmed coronavirus cases" by Facquis, used under CC BY-SA 4.0 [17]

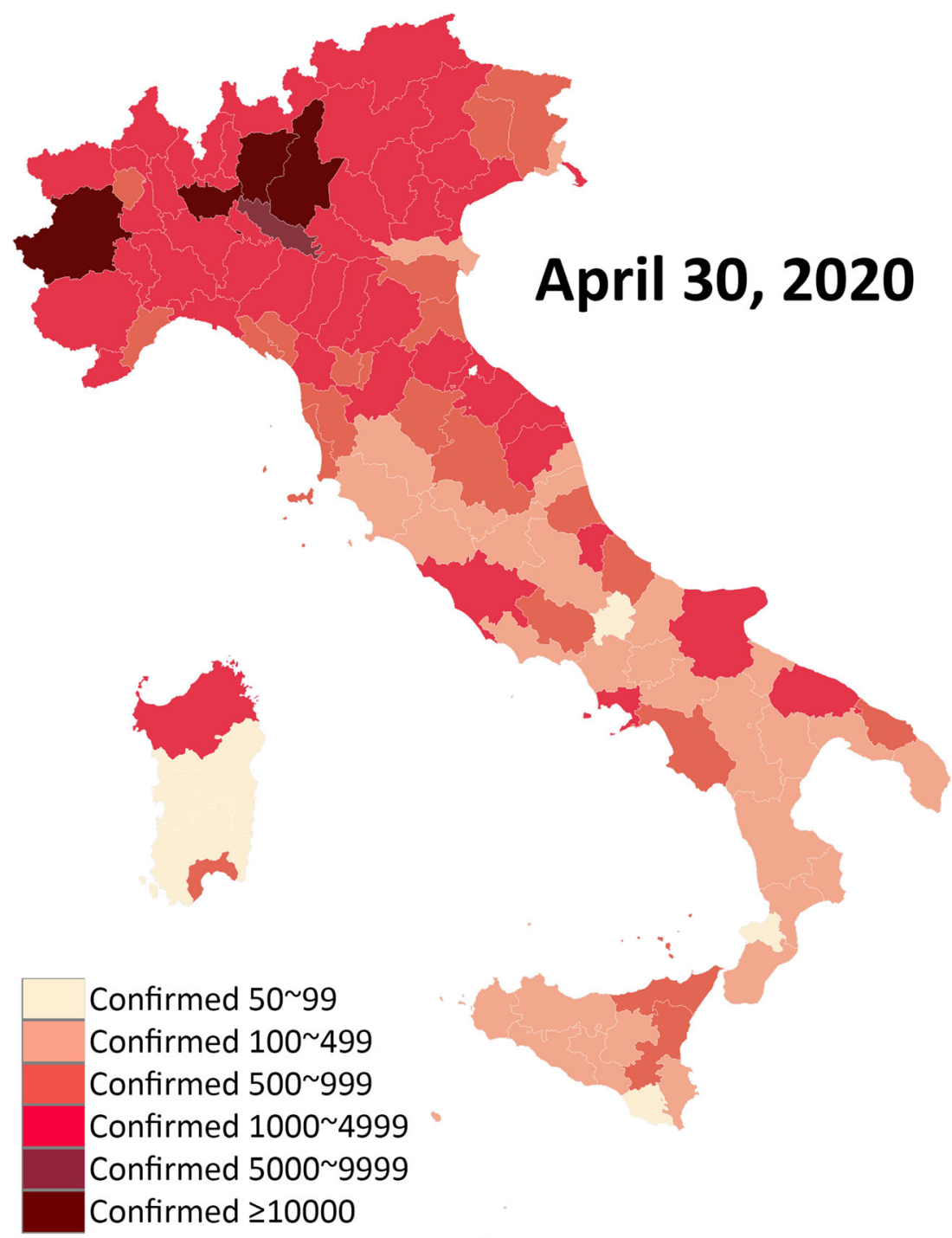




\section{Results}

A total of 2136/10,564 (20.2\%) SIRM members filled the questionnaire (mean age $47 \pm 12$ years). The majority of participants were certified radiologists $(87.7 \%$, mean age $50 \pm$ 11 years), while the remaining $12.3 \%$ were residents (mean age $30 \pm 5$ years). Geographical distribution is shown in Fig. 2.

Two-thirds of participants performed at least one diagnostic/interventional procedure on COVID-19 patients. A reduction in the elective imaging volumes was reported by $88.1 \%$ of participants. US, mammography, and MRI were the imaging techniques that suffered the greater decrease $(41.1 \%, 23.9 \%, 21.1 \%)$. In $69.6 \%$ of cases, institutions had procurement problems with personal protective equipment (PPE). Although $81.5 \%$ of participants believed that reverse transcriptase-polymerase chain reaction (RT-PCR) swab should be performed on all health workers, only $28.8 \%$ underwent the test. Almost $60 \%$ declared to work safely during the emergency, $54.6 \%$ felt to be adequately protected by their institutions, and $13.5 \%$ were redeployed to support other specialists in COVID-19 departments.

Chest US was not performed in $43 \%$ of institutions. The first imaging examination performed by admitted COVID-19 patients was chest radiography in $76.3 \%$ of cases.

Almost half of participants reported that less than $30 \%$ of health workers were infected in their radiology department.

\section{Sub-analysis according to institution type}

The $53.6 \%$ of participants work in public institutions, $26 \%$ in private institutions, and $20.3 \%$ in university hospitals. The vast majority of participants (79-83\%) agreed that RT-PCR swab should be performed on all health workers, regardless of the institution type.

As shown by answers to questions no. 3 and no. 4, private institutions performed the lowest number of examinations/ procedures on COVID-19 patients $(p<0.001)$. At the same time, private institutions were affected by the higher rate of routine working activity suspension (13.3\%), which was mainly reconverted to emergency and COVID-19-related procedures $(46.9 \%)$. Although participants from private institutions had lower PPE availability and had lower RT-PCR testing, they reported the lowest rate of infected health workers in their department, with $72.9 \%$ of participants reporting no COVID-19 infection among their colleagues. Full data regarding subgroup analysis based on institution type is reported in Supplementary Table 2.

\section{Sub-analysis according to Italian macroregions}

The majority of answers (48.7\%) came from Northern Italy, $23.8 \%$ from central regions, and $27.5 \%$ from southern Italy and Islands. Moving from the north to the south of Italy, we observed a progressive and significant decrease in the number of examinations/procedures on COVID-19 patients, as well as of the rate of infected health workers in radiology departments. Southern institutions had more trouble getting PPE (75.9\%). Chest CT was more frequently used as first imaging modality tool in southern regions $(51.3 \%)$ compared with northern (30.3\%) and central regions (44.2\%). At the same time, ultrasound was not performed in $61.9 \%$ of cases in southern regions. Full data regarding subgroup analysis based on Italian macroregions is reported in Supplementary Table 3.

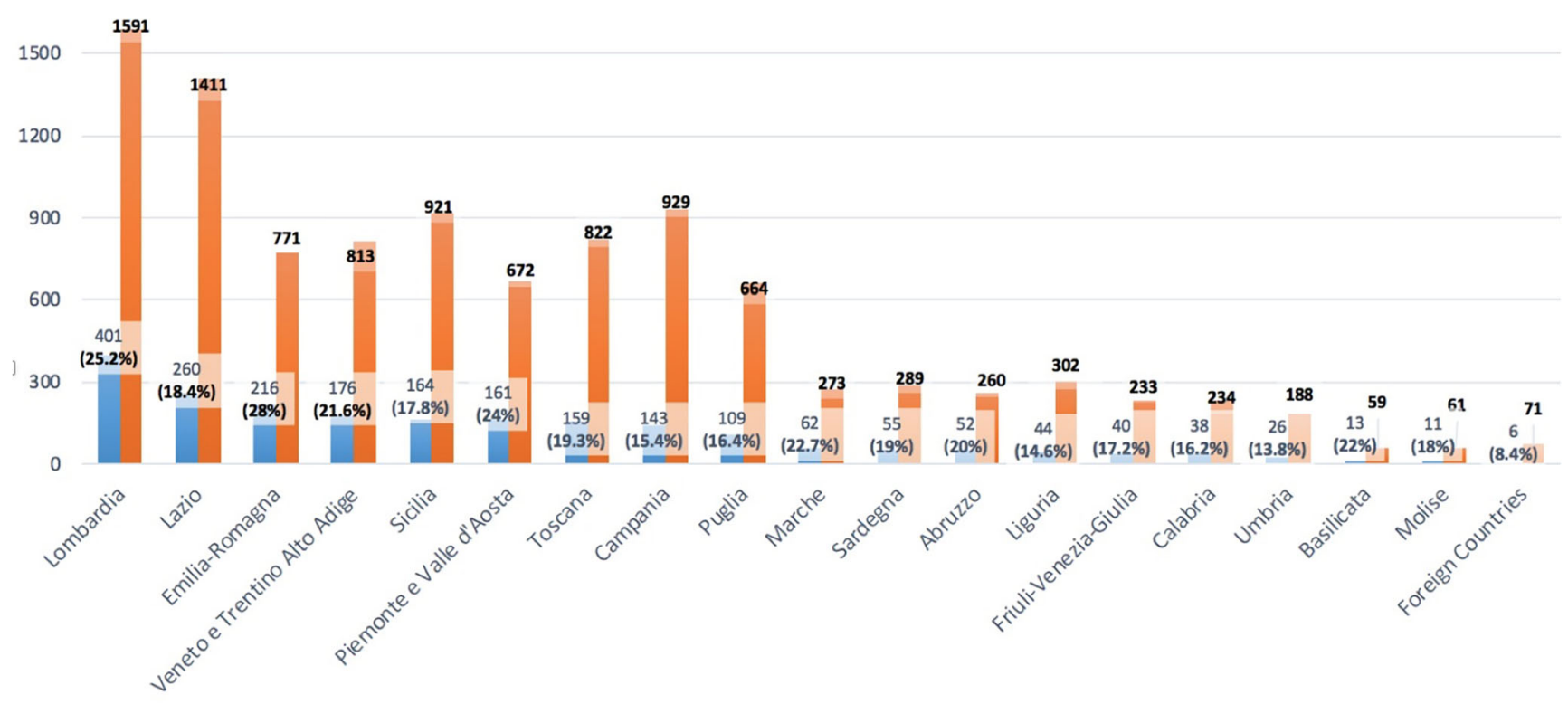

- $\mathrm{N}^{\circ}$ of answers $\quad \mathrm{N}^{\circ}$ of SIRM members

Fig. 2 Geographical distribution of survey participants with regional response rate of SIRM members (percentage in brackets) 


\section{Sub-analysis of northern Italian regions}

The northern Italian regions with the highest infection rate had no significant differences concerning the decrease of working activity ( $p=0.056$, question no. 5), supplies of PPE ( $p=$ 0.134 , question no. 9), measures adopted by radiology departments ( $p=0.293$, question no. 14), and expectations of radiologists to improve safety at work ( $p=0.109$, question no. 15). Conversely, a relevant difference was found for RTPCR testing, which was performed on $64 \%$ of participants from Veneto/Trentino-Alto-Adige (compared with 18.7$29.2 \%$ of other northern regions). Also, radiologists from Veneto/Trentino-Alto-Adige showed significantly higher satisfaction regarding their institutions' measures $(63.6 \%$ vs. $46.6-59.7 \%)$, work safety $(72.2 \%$ vs. $57.9-60.9 \%)$, as well as lower rate of infected colleagues $(p<0.001)$. Full data regarding the comparison of northern regions is reported in Supplementary Table 4.

\section{Discussion}

This paper reports the results of a nationwide survey performed to assess the impact of COVID-19 emergency on working activity of radiology departments in Italy, which was one of the countries with the most COVID-19 infections and deaths.

The analysis of our results requires a preamble about the organization of the Italian healthcare system. The national health service is administered on a regional basis, with an integration of public and private health service providers, the latter with specific contracts to provide services via the national health service. Management and organization of healthcare is delegated to each region with central recommendations provided by the Ministry of Health. During emergencies, like the ongoing COVID-19 pandemic, the activity of the National Service is supported by the Civil Protection Department, which is delegated to several subsidiary actions including the supply of PPE.

According to our data, radiology activity has dramatically changed during COVID-19 outbreak, with an extreme variability between different institutions and regions. More than two-thirds of Italian radiologists performed diagnostic/ interventional procedures on COVID-19 patients, especially those working in public and university institutions, which were indeed more involved in the emergency. A much lower number of examinations/procedures were performed on COVID-19 patients in central and southern regions, certainly due to the higher rates of infections in the north. Similarly, the rate of infected health workers in southern institutions was quite lower compared with northern, in which more than $60 \%$ of radiologists reported healthcare worker infections in their departments.
Unsurprisingly, a substantial decline in the working activity was observed in radiology departments throughout the Italian territory. As expected, this was more pronounced in private institutions where the majority of radiologists reported a precipitous decrease of elective imaging volumes, with even $13.3 \%$ of them reporting a complete suspension of imaging activity. A similar trend was observed in the USA, where the American College of Radiology suggested to reschedule nonurgent outpatient examinations, as recommended by the Center for Disease Control and Prevention [18]. In fact, suspension of outpatient imaging is considered a necessary measure to contain the spread of infection. In keeping with this objective, $70 \%$ of Italian institutions have targeted radiology activity only to emergency, inpatient, and/or COVID-19 examinations during lockdown. The impact of such "imaging lockdown" was twofold. On the one hand, we observed a tremendous reduction of crucial elective examinations related to screening programs, such as those for breast cancer [19]. According to our data, mammography showed the greatest decrease, which was second only to ultrasound. Ultrasound and interventional procedures are indeed the two radiology activities at higher risk of infection for operators [20]. On the other hand, the drop of imaging volumes was financially devastating for the economy of private institutions, which are mainly focused on outpatient imaging [21]. Furthermore, working activity of $13.4 \%$ of Italian radiologists (up to $20 \%$ in some northern regions) has been converted to support COVID-19 departments. This redeployment of radiologists to clinical services was necessary in hospital departments which were understaffed and overwhelmed by COVID-19 workload at crisis peak.

Regarding the PPE, the explosive spread of contagion in Italy made the supply of masks, gowns, visors, and goggles for eye protection a critical issue, as the importance of PPE in pandemics is well-established. Compared with public hospitals, private institutions had fewer problems getting PPE (question no. 8), probably due to their higher financial flexibility. Furthermore, southern regions had more procurement problems, probably because priority has been given for PPE supply to most affected regions. The same happened with the help of foreign countries like the USA and China that allocated medical supplies to Italy $[22,23]$.

Several measures were adopted to reduce the risk of hospital cross-infection in radiology departments. The main measures taken by institutions were the establishment of separate paths for COVID-19 patients (62\%) and clinical/temperature screening to all admitted patients and health workers $(56 \%)$, while radiology departments increased the availability of sanitizers and disinfectants (76\%), suspended the elective activity (72\%), and implemented standardized protocols of sanitation $(69 \%)$. As reported by half of participants, portable radiographic equipment and radiology machines dedicated exclusively to COVID-19 patients were also used to limit 
transportation of patients. On the other side, when outpatients were admitted to radiology department, PPE were supplied prior to their access to the department. These and many other prevention and control measures in radiology departments have been recommended by international societies and experts to support all healthcare systems that have had little time to prepare their responses to this unpredictable emergency [8, 24]. Indeed, as recently advocated by an expert panel of radiologists, radiology preparedness depends on a combination of procedures aimed at maintaining continued activity during emergency, safely ensuring diagnostic and interventional support for both patients with COVID-19 and the entirety of the hospital and health system [8]. Of note, teleradiology was overlooked as an alternative measure, probably because outsourcing has more restrictive guidelines if compared with other European countries and it has still no major role in Italian radiology departments, having gained more space in private practice [25].

RT-PCR applied to upper respiratory tract swab has been used as the preferred screening/testing technique to diagnose COVID-19 infection, although it has limited sensitivity $(60-71 \%)$ [26]. In more than two-thirds of Italian radiology departments, RT-PCR has been performed on health workers only when they presented COVID-19 symptoms or after contact with infected patients. With this strategy, less than $30 \%$ of Italian radiologists have been subjected to RTPCR swab. Of note, the vast majority of Italian radiologists believe that all health workers should be subjected to RTPCR (question no. 11) and about $70 \%$ suggest this measure as the most important to improve safety at work (question no. 15), regardless their institution or region. However, in the most affected Italian regions, this test was performed by 18-29\% of radiologists in Lombardia, Emilia-Romagna, and Piemonte/Valle d'Aosta. On the other hand, 64\% were subjected to RT-PCR swab in Veneto/Trentino-AltoAdige, where radiologists also showed higher satisfaction regarding their institutions' measures and safety at work compared with other northern regions. The strong testing policy, with aggressive contact tracing and limitation of contact with healthcare systems (through mobile diagnostic teams and home follow-up), seems to have led Veneto to better outcomes, taking advantage of a community-based approach with high integration of public health services at local levels [27]. Indeed, radiologists from Veneto/ Trentino-Alto-Adige reported a lower rate of infections among their colleagues in radiology departments. In this setting, as highlighted by a group of colleagues working in Lombardia at the epicenter of the COVID-19 emergency, this pandemic requires a shift of perspective toward community-centered care to adopt specific measures to face the spread of infection and to support the healthcare system [28]. Nevertheless, several factors played a role in the different outcomes of Lombardia: the explosive nature of the pandemic, the higher numbers of initial foci, issues related to RT-PCR kit availability, as well as higher population density [29].

In Italy, the first imaging examination performed on COVID-19 patients has been chest radiography, followed by chest CT and ultrasound. Although several studies have recently promoted the use of CT to diagnose and follow-up COVID-19 pneumonia [30, 31], this choice is probably dictated by safety issues. Indeed, CT rooms need to be thoroughly cleaned and the air needs to be recirculated to limit the risk for infection of other patients or health workers in radiology departments [32]. Of note, chest radiography proved to be useful to identify and assess the severity of COVID-19 pneumonia [33, 34]. The great majority of Italian radiologists felt confident with COVID-19 chest imaging, although $70 \%$ declared to have further broadened their knowledge. However, some concerns arose regarding chest ultrasound, especially about the importance of operator's experience in this technique, which is not routinely used in clinical practice. Indeed, only $12 \%$ of Italian radiologists have performed chest ultrasound during COVID-19 emergency, with a primary role for this technique played by emergency physicians and pneumologists.

This data should be a starting point to discuss the main criticisms arisen during the emergency, to be prepared for a possible second wave of COVID-19 infections or similar pandemics. Future health policies should be aimed at containing the spread of infection starting from the hospitals, with a better and safe organization and harmonization of the working flow to guarantee radiological inpatient and outpatient activity. As emerged by our survey, further implementation of teleradiology should be considered to split up the radiological staff into small rotating teams, with a smaller part of radiologists that would be effectively active on-site, while the remaining might work at home. This measure could be used to react to the drop of imaging volume and to prevent getting the whole team infected, especially to protect those radiologists with risk factors [35]. Furthermore, as advocated by most participants, a capillary contact and infection tracing among health workers could be another strategical way to improve safety at work and to limit cross-infection within health institutions.

This study has limitations. First, we may have not included a number of possible measures to limit the spread. Nevertheless, long and complex surveys are not attractive, so we preferred to include essential questions with higher consistency. Then, the majority of participants belonged to northern regions, with central and southern regions being relatively underrepresented. However, the response rate was only slightly higher in those regions that were more involved in the pandemic, with radiologists being probably more prone to participate. This could have introduced a possible recall bias, because participants who were more heavily involved in the 
emergency may have provided more accurate answers than those who were not in the frontline of the COVID-19 outbreak.

In conclusion, this survey offers a snapshot of the current situation in Italian radiology departments, which are involved in the frontline of the COVID-19 outbreak. Challenges and reactions of first and highly affected western countries such as Italy should serve as a model for other countries to improve the organization of radiological activity and to safely and effectively face this pandemic.

Funding information The authors state that this work has not received any funding.

\section{Compliance with ethical standards}

Guarantor The scientific guarantor of this publication is Domenico Albano.

Conflict of interest The authors of this manuscript declare no relationships with any companies, whose products or services may be related to the subject matter of the article.

Statistics and biometry No complex statistical methods were necessary for this paper.

Informed consent Informed consent was not needed as the paper does not involve patients' data directly.

Ethical approval Institutional Review Board approval was not required because the paper does not involve patients' data directly.

\section{Methodology}

- original research.

\section{References}

1. WHO. Coronavirus disease 2019 (COVID-19) Situation Report 51. https://www.who.int/docs/default-source/coronaviruse/ situation-reports/20200311-sitrep-51-covid-19.pdf?sfvrsn= 1 ba62e57 10. Accessed 2 May 2020

2. Grasselli G, Pesenti A, Cecconi M (2020) Critical care utilization for the COVID-19 outbreak in Lombardy, Italy. JAMA. https://doi. org/10.1001/jama.2020.4031

3. Colombi D, Bodini FC, Petrini M et al (2020) Well-aerated lung on admitting chest CT to predict adverse outcome in COVID-19 pneumonia. Radiology. https://doi.org/10.1148/radiol.2020201433

4. WHO. Coronavirus disease 2019 (COVID-19) Situation Report 102. https://www.who.int/docs/default-source/coronaviruse/ situation-reports/20200501-covid-19-sitrep.pdf?sfvrsn=742f4a18 2. Accessed 2 May 2020

5. Politi LS, Balzarini L (2020) The radiology department during the COVID-19 pandemic: a challenging, radical change. Eur Radiol. https://doi.org/10.1007/s00330-020-06871-0

6. Sorbello M, El-Boghdadly K, Di Giacinto I et al (2020) The Italian coronavirus disease 2019 outbreak: recommendations from clinical practice. Anaesthesia 75:724-732

7. Rubin GD, Ryerson CJ, Haramati LB et al (2020) The role of chest imaging in patient management during the COVID-19 pandemic: a multinational consensus statement from the Fleischner Society. Radiology. https://doi.org/10.1148/radiol.2020201365

8. Mossa-Basha M, Meltzer CC, Kim DC, Tuite MJ, Kolli KP, Tan BS (2020) Radiology department preparedness for COVID-19: radiology scientific expert panel. Radiology. https://doi.org/10.1148/ radiol.2020200988

9. Chen RC, Tan TT, Chan LP (2020) Adapting to a new normal? 5 key operational principles for a radiology service facing the COVID-19 pandemic. Eur Radiol. https://doi.org/10.1007/ s00330-020-06862-1

10. Remuzzi A, Remuzzi G (2020) COVID-19 and Italy: what next? Lancet 395:1225-1228

11. Giovagnoni A (2020) Facing the COVID-19 emergency: we can and we do. Radiol Med 125:337-338

12. Bignardi E, Baccaro F, De Santo D, Filippelli R, Palumbo C, Coppola M (2020) La gestione del rischio biologico da COVID19 in una UO di Radiologia https://www.sirm.org/wp-content/ uploads/2020/03/Procedure-covid19 cotugno.pdf. Accessed 2 May 2020

13. Google Forms. https://www.google.com/forms/about/. Accessed 12 Apr 2020

14. Silvestri E, Barile A, Albano D et al (2018) Interventional therapeutic procedures in the musculoskeletal system: an Italian Survey by the Italian College of Musculoskeletal Radiology. Radiol Med 123:314-321

15. Sconfienza LM, Albano D, Messina C, Silvestri E, Tagliafico AS (2018) How, when, why in magnetic resonance arthrography: an international survey by the European society of musculoskeletal radiology (ESSR). Eur Radiol 28:2356-2368

16. ISS. Coronavirus | Istituto Superiore di Sanità. ISS-Epicentro. 2020. https://www.epicentro.iss.it/en/coronavirus/. Accessed 8 May 2020

17. Facquis. Map of provinces with confirmed coronavirus cases. https://upload.wikimedia.org/wikipedia/commons/2/26/COVID19 Outbreak_Cases_in_Italy_\%28Density\%29.svg. Accessed 8 May 2020

18. ACR COVID-19 Clinical Resources for Radiologists. https://www. acr.org/Clinical-Resources/COVID-19-Radiology-Resources. Accessed 8 May 2020

19. Society of Breast Imaging Statement on Screening in a Time of Social Distancing. https://www.sbi-online.org/Portals/0/Position Statements/2020/society-of-breast-imaging-statement-on-breastimaging-during-COVID19-pandemic.pdf. Accessed 8 May 2020

20. Society of Interventional Radiology. J Vasc Interv Radiol. 2010;21(8):A5-A6 https://www.sirweb.org/practice-resources/ covid-19-resources/. Accessed 8 May 2020

21. Cavallo JJ, Forman HP (2020) The economic impact of the COVID-19 pandemic on radiology practices. Radiology. https:// doi.org/10.1148/radiol.2020201495

22. U.S. Assistance to Italy | U.S. Embassy Consulates in Italy. https:// it.usembassy.gov/u-s-assistance-to-italy/. Accessed 8 May 2020

23. Coronavirus: Chinese aid to the EU delivered to Italy. https://ec. europa.eu/commission/presscorner/detail/en/IP 20_600. Accessed 8 May 2020

24. Ding J, Fu H, Liu Y et al (2020) Prevention and control measures in radiology department for COVID-19. Eur Radiol. https://doi.org/ 10.1007/s00330-020-06850-5

25. Coppola F, Bibbolino C, Grassi R et al (2016) Results of an Italian survey on teleradiology. Radiol Med 121:652-659

26. Yang Y, Yang M, Shen C et al (2020) Evaluating the accuracy of different respiratory specimens in the laboratory diagnosis and monitoring the viral shedding of 2019-nCoV infections. medRxiv. Cold Spring Harbor Laboratory Press. https://doi.org/10.1101/ 2020.02.11.20021493v2

27. Binkin N, Salmaso S,Michieletto F, Russo F (2020) Protecting our health care workers while protecting our communities during the COVID-19 pandemic: a comparison of approaches and early 
outcomes in two Italian regions, Italy, 2020. medRxiv. Cold Spring Harbor Laboratory Press; 2020;2020.04.10.20060707. https://doi. org/10.1101/2020.04.10.20060707v2. Accessed 8 May 2020

28. Nacoti M, Ciocca A, Giupponi A et al (2020) At the epicenter of the Covid-19 pandemic and humanitarian crises in Italy: changing perspectives on preparation and mitigation. Catal non-issue content 2020;1(2):1-5. https://catalyst.nejm.org/doi/full/10.1056/CAT.20. 0080. Accessed 8 May 2020

29. Pisano GP, Sadun R, Zanini M (2020) Lessons from Italy's response to coronavirus. Harv Bus Rev 2020;1-12. https://hbr.org/ 2020/03/lessons-from-italys-response-to-coronavirus. Accessed 8 May 2020

30. Caruso D, Zerunian M, Polici M et al (2020) Chest CT features of COVID-19 in Rome, Italy. Radiology. https://doi.org/10.1148/ radiol.2020201237

31. Pan F, Ye T, Sun P et al (2020) Time course of lung changes on chest CT during recovery from 2019 novel coronavirus (COVID19) pneumonia. Radiology. https://doi.org/10.1148/radiol. 2020200370
32. Hope MD, Raptis CA, Henry TS (2020) Chest computed tomography for detection of coronavirus disease 2019 (COVID-19): Don't rush the science. Ann Intern Med. https://doi.org/10.7326/M201382

33. Borghesi A, Zigliani A, Masciullo R et al (2020) Radiographic severity index in COVID-19 pneumonia : relationship to age and sex in 783 Italian patients. Radiol Med. https://doi.org/10.1007/ s11547-020-01202-1

34. Bandirali M, Sconfienza LM, Serra R et al (2020) Chest radiograph findings in asymptomatic and minimally symptomatic quarantined patients in Codogno, Italy during COVID-19 pandemic. Radiology 295:E7

35. Mossa-Basha M, Medverd J, Linnau K et al (2020) Policies and guidelines for COVID-19 preparedness: experiences from the University of Washington. Radiology. https://doi.org/10.1148/ radiol.2020201326

Publisher's note Springer Nature remains neutral with regard to jurisdictional claims in published maps and institutional affiliations.

\section{Affiliations}

\section{Domenico Albano ${ }^{1,2}$ (D) Antonio Bruno ${ }^{3}$ - Federico Bruno ${ }^{4} \cdot$ Marco Calandri $^{5,6}$. Damiano Caruso ${ }^{7}$. Alfredo Clemente ${ }^{8} \cdot$ Pietro Coppolino $^{9} \cdot$ Diletta Cozzi $^{10} \cdot$ Riccardo De Robertis $^{11}$ • Francesco Gentili ${ }^{12}$. Irene Grazzini ${ }^{13}$. Maria Laura Jannone ${ }^{14}$. Carlo Liguori ${ }^{15} \cdot$ Raffaele Natella $^{8} \cdot$ Genny Pace $^{16}$. Alessandro Posa ${ }^{17}$. Paola Scalise ${ }^{18}$. Bruno Accarino ${ }^{19} \cdot$ Corrado Bibbolino $^{20} \cdot$ Antonio Barile $^{4} \cdot$ Roberto Grassi $^{8} \cdot$ Carmelo Messina $^{1,21} \cdot$ On behalf of the Young SIRM Working Group}

1 IRCCS Istituto Ortopedico Galeazzi, Via R. Galeazzi 4, 20161 Milan, Italy

2 Department of Biomedicine, Neurosciences and Advanced Diagnostics, Section of Radiological Sciences, University of Palermo, Palermo, Italy

3 Radiology department, SS. Annunziata Hospital, Cento (Fe), Italy

4 Department of Biotechnology and Applied Clinical Sciences, University of L'Aquila, L'Aquila, Italy

5 Department of Oncology, Radiology Unit, University of Torino, Turin, Italy

6 Radiology Unit, A.O.U. San Luigi Gonzaga - Orbassano (To), Orbassano, TO, Italy

7 Department of Radiological, Oncological and Pathological Sciences, "Sapienza" University of Rome, Sant'Andrea University Hospital, Rome, Italy

8 Department of Precision Medicine, University of Campania "L. Vanvitelli", Naples, Italy

9 Department of Medical Surgical Sciences and Advanced Technologies, "G.F. Ingrassia"-Radiology I Unit, University Hospital "Policlinico-Vittorio Emanuele", Catania, Italy

10 Department of Emergency Radiology, University Hospital Careggi, Largo Brambilla 3, 50123 Florence, Italy
11 Department of Radiology, Ospedale Civile Maggiore, Azienda Ospedaliera Universitaria Integrata Verona, Verona, Italy

12 Department of Medicine and Surgery, Unit of Radiology, Azienda Ospedaliera Universitaria di Parma, Parma, Italy

13 Department of Radiology, Section of Neuroradiology, San Donato Hospital, Arezzo, Italy

14 Università degli Studi di Milano, Milan, Italy

15 Department of Radiology, Ospedale del Mare-ASLNa1 Centro, Naples, Italy

16 Radiology Unit, Humanitas Mater Domini, Castellanza, VA, Italy

17 Department of Diagnostic Imaging, Oncology, Radiotherapy and Hematology, Fondazione Policlinico Universitario Agostino Gemelli - IRCCS, Rome, Italy

18 Department of Diagnostic Imaging, Pisa University Hospital, Via Paradisa 2, 56124 Pisa, Italy

19 SIRM, Resources Management Study Group, Milan, Italy

20 SNR Foundation, Rome, Italy

21 Dipartimento di Scienze Biomediche per la Salute, Università degli Studi di Milano, Milan, Italy 